\title{
Eficacia del proyecto de creación del Centro Deportivo de la Fuerza Aérea desde la perspectiva de las partes interesadas*
}

\author{
Creation project effectiveness of the Air Force Sports Center in the perspective \\ of stakeholders
}

\begin{abstract}
Eficácia do projeto de criação do Centro de Desportos da Aeronáutica na perspectiva dos stakeholders
\end{abstract}

Cel Inf Pedro Celso Gagliardi Palermo, Maestro Comisión de Deportes de la Fuerza Aérea - CDA

Rio de Janeiro/RJ - Brasil pcgagliardi@gmail.com

\section{RESUMEN}

El estudio tuvo por objeto analizar la forma en que los factores críticos de éxito, desde el punto de vista de las partes interesadas, ejercen impacto sobre la eficacia del proyecto de implantación del Centro de Deportes de la Fuerza Aérea (CDAer). Se identificó a los principales actores involucrados en el proceso y se los sometió a una investigación por medio de un cuestionario. El instrumento se basó en los factores críticos de éxito propuestos por Pinto y Slevin (1987). Las preguntas se adaptaron a la realidad de la propuesta con el fin de permitir el análisis del grado de importancia de cada factor crítico, así como la eficacia estratégica y táctica del proyecto. Los resultados mostraron que la mayoría de las partes interesadas consideran que el proyecto está en el buen camino y tiene una eficacia más estratégica que táctica, sin embargo, algunos necesitan recibir más atención para que se satisfagan sus expectativas. La metodología aplicada también permitió señalar posibles errores que pueden ocurrir en el desarrollo de la implantación del CDAer, que deben ser mejor administrados. En conclusión, se sugiere la realización de un diagnóstico claro de sus problemas, especialmente de los relacionados con los clientes, el personal, los recursos financieros y la estructura orgánica, respetando el equilibrio entre la eficacia estratégica y táctica de la propuesta, con el fin de satisfacer a todos los interesados.

Palabras clave: Evaluación de las partes interesadas. Deporte militar. Gestión deportiva. Eficacia estratégica y táctica.

Recibido / Received / Recebido $10 / 09 / 14$
Aceptado / Accepted / Aceito $22 / 07 / 15$

*Este estudio constituye el Trabajo Final (TCC), dirigido por el profesor Ricardo de Moura, del Curso Superior en Gestión Deportiva (CAGE), 2013-2014, dictado por el Instituto Olímpico Brasileño (IOB), vinculado al Comité Olímpico Brasileño (COB) y a Solidaridad Olímpica. 


\begin{abstract}
This study aimed at analyzing how the critical success factors in the optics of its stakeholders, impact the effectiveness of the deployment project of the Air Force Sports Centre (CDAer). The main actors involved in the process were identified and subjected to an investigation through a questionnaire. The instrument was based on the critical success factors proposed by Pinto and Slevin (1987). The questions were adapted to the reality of the proposal in order to allow analysis of the degree of importance of each critical and strategic effectiveness and project tactics. The results showed that most of the stakeholders understand that the project follows the right track and has a high strategic rather than tactical effectiveness, but some need to receive more attention so that their expectations are met. The methodology enabled even to point out potential errors that may occur in the implementation of the CDAer and that they should be better managed. In conclusion, we suggest that a clear diagnosis of their problems is to be carried out, especially those related to customers, personnel, financial resources and the organizational structure, respecting the balance between the strategic and tactical effectiveness of the proposal in order to cater to all interested parties.
\end{abstract}

Keywords: Stakeholder assessments. Military sport. Sports management. Strategic and tactical effectiveness.

\title{
RESUMO
}

O presente estudo teve por objetivo analisar de que forma os fatores críticos de sucesso, na ótica dos seus stakeholders, impactam na eficácia do projeto de implantação do Centro de Desportos da Aeronáutica (CDAer). Os principais atores envolvidos no processo foram identificados e submetidos a uma investigação por meio de questionário. O instrumento teve por base os fatores críticos de sucesso propostos por Pinto e Slevin (1987). As perguntas foram adaptadas à realidade da proposta, de forma a permitir a análise do grau de importância de cada fator crítico, bem como a eficácia estratégica e tática do projeto. Os resultados demonstraram que a maior parte dos stakeholders entendem que o projeto segue um bom caminho e apresenta uma eficácia estratégica maior que tática, no entanto alguns necessitam receber maior atenção para que suas expectativas sejam atendidas. $A$ metodologia empregada possibilitou ainda apontar os erros potenciais que podem ocorrer no desenvolvimento da implantação do CDAer e que devem ser melhor gerenciados. Como conclusão, sugere-se que seja realizado um claro diagnóstico de seus problemas, principalmente dos relacionados aos clientes, ao pessoal, aos recursos financeiros e à estrutura organizacional, respeitando-se o balanceamento entre a eficácia estratégica e tática da proposta, a fim de atender a todos os interessados.

Palavras-chave: Avaliação de stakeholders. Esporte militar. Gestão esportiva. Eficácia estratégica e tática.

\section{INTRODUCCIÓN}

Con la llegada de los Juegos Olímpicos 2016, junto con la celebración de diversos eventos deportivos de gran magnitud, el deporte brasileño, incluido el segmento militar, ha alcanzado un nuevo nivel de desarrollo.

Fruto de los V Juegos Militares Mundiales del Consejo Internacional de Deportes Militares (CISM), que tuvieron lugar en Río de Janeiro en 2011, el deporte militar se considera cada vez más como un importante desarrollador del talento deportivo en el país, y cuenta en sus filas con un gran número de atletas de nivel mundial, en una diversidad de deportes.

El Comando de la Fuerza Aérea (COMAER), a través de la Comisión de Deportes de la Fuerza Aérea (CDA), está estudiando la transformación de esta unidad en el Centro Deportivo de la Fuerza Aérea (CDAer). El proyecto tiene como objeto satisfacer la demanda interna de la Fuerza Aérea relativa a la preparación física y profesional de sus militares para llevar a cabo sus actividades profesionales, sobre todo las de combate, y contribuir a los esfuerzos del país en el desarrollo del deporte de alto rendimiento, para la participación de los atletas brasileños en los Juegos Olímpicos.

Desde su creación, la estructura física y organizativa de la CDA ha cambiado poco y en la actualidad no logra responder satisfactoriamente a todas sus demandas. La falta de personal calificado en investigación, infraestructura inadecuada y dificultad para satisfacer a todas las unidades militares del COMAER impiden que los militares de la Fuerza Aérea y los atletas de alto rendimiento reciban el apoyo técnico-científico y logístico-administrativo adecuado, para ayudarlos a mejorar su rendimiento.

Además, las restricciones presupuestarias para la inversión de recursos financieros en actividades distintas de las directamente relacionadas con la 
actividad principal de la Fuerza Aérea, obstaculizan el avance de la organización en términos estructurales, lo que invariablemente dificulta una actuación integral de la CDA dentro del COMAER.

Con la llegada de los Juegos Olímpicos al país, el Ministerio de Deportes está invirtiendo en la reestructuración y modernización de las instalaciones deportivas de la CDA, con la construcción de un moderno equipamiento que será utilizado por los equipos extranjeros durante el período de preparación para los Juegos Olímpicos 2016.

Los recursos para tal fin, provistos por el Ministerio de Deportes, además de responder a las demandas olímpicas, transformarán el campus de la CDA en un moderno centro deportivo. Se espera que este legado, que después de los Juegos quedará bajo la responsabilidad del COMAER, pueda administrarse de modo de satisfacer las necesidades tanto de la Fuerza Aérea como del deporte nacional.

El apoyo financiero del Ministerio de Deportes resuelve el problema financiero de la reestructuración de la infraestructura de la Organización, pero es necesario entender cómo se llevarán adelante los procesos de gestión para satisfacer a las partes interesadas de diferentes naturalezas.

Conocer las demandas y satisfacer a las partes interesadas es la clave para la supervivencia de una organización (FREEMAN, 1984). Esta declaración expresa, directamente, la importancia de los mayores interesados en el éxito de un proyecto en particular. Y, en el caso de la creación del CDAer, no es diferente.

Por consiguiente, el problema surge de la siguiente inquietud: ¿el CDAer está siendo diseñado para satisfacer las demandas que se esperan de cada grupo de interés?

El objetivo general de este estudio es analizar la forma en que los factores críticos de éxito, desde el punto de vista de las partes interesadas, ejercen impacto sobre la eficacia del proyecto de implantación del CDAer. Por otra parte, los objetivos específicos son:

1. identificar las principales partes interesadas involucradas en el proyecto de creación del CDAer;

2. adecuar los factores críticos de éxito a las particularidades del proyecto de creación del CDAer, según la propuesta de Pinto y Slevin (2007).

3. identificar cómo las partes interesadas perciben el grado de importancia de los factores críticos de éxito (FCS ) relativos al proceso de creación del CDAer, y

4. analizar, según el grado de importancia atribuido por las partes interesadas a los FCS, la eficacia del proyecto y sus errores latentes.
El estudio se justifica por la profundización de la cita de Motta (2012), en la que el autor llega a la conclusión de que, a partir de fines del siglo XX, las teorías de administración se centran cada vez más en la satisfacción de los deseos de los consumidores, ya que ellos orientan los esfuerzos y recursos de las organizaciones eficaces.

\section{FUNDAMENTO TEÓRICO}

Según Santos (2008), el análisis de las partes interesadas contribuye a la formulación de una estructura lógica que ayuda a la gestión del proyecto a identificar estrategias adecuadas y monitorear los posibles conflictos de interés.

De acuerdo con Goldschimidt (2007), las necesidades y expectativas de cada grupo varían y es importante que cada uno de ellos perciba que sus necesidades y expectativas se están satisfaciendo.

Con el fin de cumplir o superar esas expectativas, Rabechini (2007) sugiere la aplicación de un sistema de gestión de las partes interesadas. En este sentido, el autor muestra que la gestión de las partes interesadas requiere investigar las necesidades e implementar acciones dirigidas a la satisfacción de las expectativas, tal como se describe en la Figura 1.

Figura 1 - Modelo de gestión de las partes interesadas.

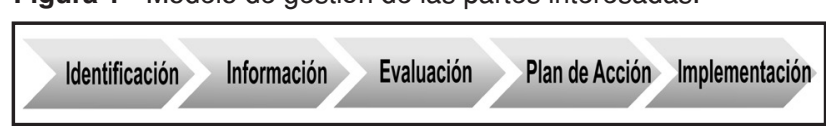

Fuente: Rabechini (2007, p. 26).

Para identificar a los interesados, el autor propone una subdivisión de las partes interesadas en primarias y secundarias. Las primarias son: proveedores, patrocinadores, acreedores, entorno local, gerente de proyecto, gerente funcional, contratistas y consumidores. Por su parte, las secundarias son: organizaciones sociales y políticas, competidores, comunidad, público, turistas, medios de comunicación, miembros de la familia y ecologistas.

Santos (2008) recomienda que, al nombrar las partes interesadas, no se puede ser demasiado específico, ni es esencial conectar unos con otros a todos los actores existentes. Por lo tanto, por razones prácticas, puede ser útil tener en cuenta grupos y subgrupos dentro de las organizaciones, y reducir la lista inicial a aquellos cuyos intereses comunes son más relevantes.

Qualman (1997) señala que algunos actores tienen mayor poder y pueden ejercer más influencia sobre el diseño, la implementación y los resultados esperados de un proyecto dado, y los clasifica de acuerdo a la matriz que se muestra en el Cuadro 1. 
Cuadro 1 - Matriz de partes interesadas.

\begin{tabular}{|c|c|}
\hline $\begin{array}{l}\text { C- Bajo interés, alto poder }(-/+) \\
\text { Estos actores pueden influir en los resultados del } \\
\text { proyecto, aunque sus prioridades no sean las mismas } \\
\text { que las del proyecto. Como son un riesgo u obstáculo, } \\
\text { deben ser estrechamente monitoreados. }\end{array}$ & $\begin{array}{l}\text { A- Alto interés, alto poder }(+/+) \\
\text { Son los actores más importantes. En este sentido, debe } \\
\text { hacerse todo lo posible para que estén satisfechos con } \\
\text { el progreso del proyecto. }\end{array}$ \\
\hline $\begin{array}{l}\text { D- Bajo interés, bajo poder (-/-) } \\
\text { Este grupo debe ser monitoreado para saber si habrá } \\
\text { cambios en relación a su interés o poder. Aunque } \\
\text { aparentemente presente una menor importancia, no } \\
\text { debe ser descuidado. }\end{array}$ & $\begin{array}{l}\text { B- Alto interés, bajo poder (+/-) } \\
\text { Este grupo es muy útil para proporcionar ideas y } \\
\text { ayudar con acciones menores. Habrá de mantenerlo } \\
\text { informado y requerirá iniciativas especiales para } \\
\text { proteger sus intereses. }\end{array}$ \\
\hline
\end{tabular}

Fuente: Adaptado de Qualman (1997).

Robinson et al. (2007), cuando se refieren específicamente a las partes interesadas de organizaciones deportivas olímpicas (OEO), dicen que normalmente los aspectos financieros del proyecto determinan el grado de interés y el poder (influencia) de cada grupo de interés en el proyecto, pero que no son los únicos. También señalan que los aspectos culturales intrínsecos interfieren en la distribución de los recursos asignados, lo que ayuda a explicar determinadas decisiones y por qué ciertos grupos parecen más importantes que otros.

Según el estudio, el aspecto cultural implica el uso del poder para dirigir, controlar y regular las actividades de una organización en lo que se conoce como Gobierno Corporativo. Estas reglas definen el ámbito de actuación de los directores, cómo se los controla y qué decisiones deben tomarse para la participación de todos los interesados legítimos.

Esto es particularmente importante para el CDAer, puesto que el proyecto de su creación implica instituciones insertadas en diferentes contextos culturales, lo que da lugar a diferentes intereses que necesitan ser contemporizados desde el nacimiento del proyecto.

Para ello, tiene que haber un equilibrio adecuado en la toma de decisiones, que debe ser respaldada por los objetivos fijados por la organización y alineada con los resultados esperados por las partes interesadas.

Santos (2008) informa de que el análisis de las partes interesadas ayuda desde la etapa de diseño hasta el desarrollo del marco lógico del proyecto, y permite identificar correctamente la participación de cada una de ellas. También ayuda a anticipar las objeciones y permite proponer medidas adecuadas para superarlas. El tipo de enfoque de la gestión de las relaciones con los actores dependerá, entre otras cosas, de la situación, el grado de dificultad en la obtención de información y la facilidad en alcanzar a los actores involucrados. Son éstos, según el autor, los factores críticos en la elección del método de análisis de las partes interesadas.

Por lo general, un proyecto está liderado por un gerente de proyecto que, en última instancia, es uno los principales responsables de su éxito. Sin embargo, Slevin y Pinto (1987) dan gran importancia tanto a los gestores como a las otras partes interesadas, y resaltan que todos los grupos deben evaluar periódicamente el progreso del proyecto y, además, brindar ideas desde varios puntos de vista, a fin de reforzar los objetivos y dar su impresión sobre el cumplimiento de sus expectativas.

Los autores proponen 10 (diez) factores críticos que se pueden utilizar en la investigación e identificación de los riesgos potenciales que afectan el éxito de cualquier proyecto, a saber: misión, apoyo de la dirección, planificación, cliente consultor, personal, asuntos técnicos, cliente aceptado, monitoreo, comunicación y reconciliación. Los tres primeros factores críticos - misión, apoyo de la dirección y planificación - se refieren a las estrategias del proyecto, es decir, al proceso de establecer los objetivos generales y lo que se quiere conseguir con ellos. Los otros factores están más relacionados con los aspectos tácticos, la acción y el logro de los objetivos establecidos.

Los autores también establecen 4 (cuatro) fases de un proyecto durante su ciclo de vida: diseño, planificación, ejecución y finalización. El nivel de esfuerzo del equipo y de las partes interesadas crece desde el diseño, a través de la planificación, hasta la ejecución del proyecto. En esta tercera fase, alcanza su ápice y, luego, disminuye hasta la fase de finalización. En las dos primeras etapas - diseño y planificación - prevalece estrategia, y las fases de ejecución y finalización, la táctica. Al final, ambos aspectos - estrategia y táctica - tienen prácticamente el mismo nivel de importancia para el éxito del proyecto (SLEVIN; PINTO, 1987).

Los autores concluyen que: a) el uso del modelo de múltiples factores es viable cuando se quiere entender el camino que se está siguiendo en un proyecto determinado; b) estos factores deben ser considerados estratégicamente desde el principio, ya que son muy sensibles para el éxito de la implementación propuesta; c) la gestión del proyecto y las partes interesadas deben desarrollar acciones estratégicas y tácticas, teniendo en cuenta el equilibrio y la transición de importancia entre los factores críticos que los definen; y d) a medida que el ciclo de vida avanza, se debe pensar y actuar tácticamente.

Además de los factores económicos y culturales mencionados, otras variables de naturaleza mercadológica 
también afectan la planificación estratégica e influyen en la gestión de una OEO.

Según Rocha y Bastos (2011), la actividad principal de la gestión de una OEO es la producción y el marketing de servicios relacionados con los deportes. La producción se refiere a la transformación de la materia prima en el producto final, mientras que las ofertas de marketing deportivo, a todas las actividades destinadas a satisfacer las necesidades y deseos de los consumidores de deportes a través de procesos de intercambio (MULLIN; HARDY; SUTTON, 2007 apud ROCHA; BASTOS, 2011).

Motta (2012) dice que el producto de una arena deportiva, como la que será el CDAer después de la finalización de las obras de infraestructura, se caracteriza por aspectos tangibles e intangibles, a saber:

- tangibles: instalaciones y equipamiento, muebles, equipo funcional;

- intangibles: apelaciones emocionales, aspectos culturales, valores institucionales.

En el caso de CDAer, la inversión de fondos públicos en una instalación militar es vista como una garantía de que los recursos se están empleando en una organización creíble. Toledo (2013) afirma que las Fuerzas Armadas se encuentran entre las instituciones que tienen una de las tasas más altas de la confianza social. En este sentido, si bien los recursos para la modernización de las instalaciones deportivas (producto tangible) son financiados por el Ministerio de Deportes, los valores cívicos e institucionales, conjuntamente con los aspectos culturales (producto intangible) de las organizaciones militares, implican el entendimiento de que, de alguna manera, ya sea por las medallas a ganar o por la mejor preparación de los militares en la defensa nacional, siempre habrá un retorno justificable para la sociedad.

Según Rabechini (2007), el éxito o fracaso de la implementación de un proyecto depende de la correcta comprensión de los conceptos y requisitos de su gestión, sobre todo cuando se trata de adecuar los deseos de los asociados a intereses aparentemente difusos. En este caso, hay que identificar y maximizar las sinergias potenciales existentes entre ellos, con el fin de favorecer la adopción de una amplia planificación capaz de generar resultados concretos para los involucrados.

Dicho esto, adquiere importancia la aplicación de un método de investigación y control de los factores críticos de éxito, ya que, a través de su correcta comprensión, se puede anticipar la identificación de posibles errores y proponer las debidas recomendaciones y correcciones del curso del proyecto.

\section{METODOLOGÍA}

Para la identificación de las partes interesadas, se siguió el método propuesto por Rabechini (2007) y la técnica de dinámica de grupo, conocido como brainstorming, entre los Oficiales de la CDA que redactaron la Propuesta de
Constitución del CDAer (BRASIL, 2015). Después de la determinación de los actores primarios y secundarios, se determinaron los 4 (cuatro) grupos principales, que se describen como sigue:

G1 - Oficiales Generales miembros del Alto Comando de la Fuerza Aérea, Secretario Nacional de Deportes de Alto Rendimiento del Ministerio de Deportes, presidente de la CDA y presidente de la Comisión Deportiva Militar de Brasil (CDMB).

G2 - Oficiales de alto rango del Departamento de Educación de la Fuerza Aérea, del Estado Mayor de la Fuerza Aérea, del Comando General de Personal, del Centro de Control Interno de la Fuerza Aérea, además de los gestores del proyecto de la CDA.

G3 - Presidentes o directores técnicos de las organizaciones deportivas civiles y militares con las que la CDA tiene una relación directa, asesores de la Secretaría de Deportes de Alto Rendimiento del Ministerio de Deportes y miembros del Comité Olímpico Brasileño.

G4 - Militares de carrera de la Fuerza Aérea, atletas civiles y militares y secciones de Educación Física de las escuelas militares.

En este estudio se adaptaron los factores críticos propuestos por Slevin y Pinto (1987) y se adoptó la sugerencia de Santos (2008), de que el análisis de las partes interesadas dependerá de la situación, el grado de dificultad en la obtención de información y la facilidad en alcanzar a los actores involucrados.

En la elaboración del cuestionario, se tuvo en cuenta el contenido del documento titulado Proposta de Criação do CDAer [Propuesta de Constitución del CDAer] (BRASIL, 2015). Sobre esta base, se propusieron 45 (cuarenta y cinco) preguntas divididas en 9 (nueve) bloques de 5 (cinco) preguntas cada uno. Las opciones de respuesta variaron, según la escala de Likert, de 1 (uno) a 7 (siete), en la que 1 (uno) significa "No estoy de acuerdo en absoluto" y 7 (siete), "Estoy completamente de acuerdo".

Se validó el cuestionario a través de una prueba preliminar, según las indicaciones de Marconi y Lakatos (2003, p. 203), a través de la evaluación de 4 (cuatro) oficiales superiores y 1 (uno) director técnico de Confederación Deportiva, quienes sugirieron las adaptaciones necesarias para su perfecta comprensión.

El cuestionario se envió a 66 (sesenta y seis) interesados, representantes de los 4 (cuatro) grupos identificados. Para la inclusión en el estudio, se decidió seleccionar a los que tuvieran conocimiento de la propuesta de creación del CDAer, aunque con distintos grados de profundidad. Como el interés y el poder de cada grupo de interés es diferente, se seleccionaron las preguntas planteadas a cada uno de ellos, de modo tal que tuvieran que responder únicamente las que les resultaran más afines.

El Cuadro 2 muestra la distribución de las preguntas por grupo de actores involucrados y la cantidad de cuestionarios distribuidos. 
Cuadro 2 - Distribución de las preguntas por grupo de interés.

\begin{tabular}{|c|c|c|c|c|}
\hline $\begin{array}{c}\text { Preguntas relativas a los } \\
\text { factores críticos }\end{array}$ & G1 & G2 & G3 & G4 \\
\hline $\begin{array}{c}\text { FCE } 1 \text { - Objetivos centrales del } \\
\text { CDAer }\end{array}$ & $\mathrm{x}$ & $\mathrm{x}$ & $x$ & $x$ \\
\hline $\begin{array}{c}\text { FCE } 2 \text { - Dirección del } \\
\text { COMAER } \\
\end{array}$ & $\mathrm{x}$ & $\mathrm{x}$ & & \\
\hline $\begin{array}{c}\text { FCT } 3 \text { - Estructura orgánica } \\
\text { propuesta }\end{array}$ & $x$ & $\mathrm{x}$ & $x$ & $x$ \\
\hline $\begin{array}{c}\text { FCT } 4 \text { - Preguntas relativas al } \\
\text { personal } \\
\end{array}$ & $\mathrm{x}$ & $\mathrm{x}$ & & \\
\hline $\begin{array}{c}\text { FCT } 5 \text { - Recursos financieros } \\
\text { para la vida vegetativa }\end{array}$ & $\mathrm{x}$ & $\mathrm{x}$ & & \\
\hline FCE 6 - Planificación & & $\mathrm{x}$ & & \\
\hline FCT 7 - Infraestructura & & $\mathrm{x}$ & $\mathrm{x}$ & $\mathrm{x}$ \\
\hline $\begin{array}{l}\text { FCT } 8 \text { - Cliente (potenciales } \\
\text { beneficiarios del CDAer) }\end{array}$ & & $\mathrm{x}$ & $\mathrm{x}$ & $\mathrm{x}$ \\
\hline FCE 9 - Apoyo al proyecto & & $\mathrm{x}$ & $\mathrm{x}$ & $\mathrm{x}$ \\
\hline $\begin{array}{c}\text { Cantidad de cuestionarios } \\
\text { enviados } \\
\end{array}$ & 6 & 11 & 21 & 28 \\
\hline $\begin{array}{l}\text { Cantidad y porcentaje de } \\
\text { cuestionarios devueltos }\end{array}$ & $\begin{array}{c}1 \\
(16 \%)\end{array}$ & $\begin{array}{c}6 \\
(54 \%)\end{array}$ & $\begin{array}{c}13 \\
(61 \%)\end{array}$ & $\begin{array}{c}14 \\
(50 \%)\end{array}$ \\
\hline
\end{tabular}

Legenda: FCE: factor crítico estratégico; FCT: factor crítico táctico. Fuente: El autor.

Una vez devueltos los cuestionarios, se llevó a cabo la identificación de las modas y los valores mínimos de cada respuesta por factor crítico y por grupo de interesados. Se utilizó la moda porque es una medida de tendencia central que representa el valor más frecuente. Los valores mínimos, a su vez, representan las opiniones más negativas, que, una vez conocidas, pueden agregar valor en la proposición de recomendaciones para las correcciones del curso del proyecto.

A continuación, se hizo la sumatoria de las modas y los valores mínimos y se la correlacionó con los rangos porcentuales (Cuadro 3), sobre una adaptación de la propuesta de Pinto y Slevin (1998 apudRABECHINI, 2007).

Cuadro 3 - Rangos porcentuales de los factores críticos de éxito.

\begin{tabular}{|c|c|c|c|c|c|c|c|c|c|}
\hline Porcentaje & FCE & FCE & FCT & FCT & FCT & FCE & FCT & FCT & FCE \\
& $\mathbf{2}$ & $\mathbf{3}$ & $\mathbf{4}$ & $\mathbf{5}$ & $\mathbf{6}$ & $\mathbf{7}$ & $\mathbf{8}$ & $\mathbf{9}$ \\
\hline $100 \%$ & 35 & 35 & 35 & 35 & 35 & 35 & 35 & 35 & 35 \\
\hline $90 \%$ & 34 & 34 & 34 & 32 & 32 & 33 & 32 & 34 & 34 \\
\hline $80 \%$ & 33 & 32 & 32 & 30 & 30 & 31 & 30 & 33 & 32 \\
\hline $70 \%$ & 32 & 30 & 31 & 28 & 28 & 30 & 28 & 32 & 30 \\
\hline $60 \%$ & 31 & 28 & 30 & 27 & 27 & 28 & 27 & 31 & 28 \\
\hline $50 \%$ & 30 & 27 & 29 & 24 & 24 & 27 & 24 & 30 & 27 \\
\hline $40 \%$ & 29 & 25 & 27 & 22 & 22 & 26 & 22 & 29 & 25 \\
\hline $30 \%$ & 28 & 23 & 26 & 20 & 20 & 24 & 20 & 27 & 23 \\
\hline $20 \%$ & 26 & 20 & 24 & 18 & 18 & 21 & 18 & 25 & 20 \\
\hline $10 \%$ & 25 & 17 & 21 & 14 & 14 & 16 & 14 & 22 & 17 \\
\hline $0 \%$ & 7 & 6 & 8 & 5 & 5 & 5 & 5 & 7 & 6 \\
\hline
\end{tabular}

Fuente: Adaptado de Pinto y Slevin (1998 apud RABECHINI, 2007, p. 54).
Los factores con un buen rendimiento son aquellos en el rango entre el 80 y $100 \%$. Los factores situados entre el 50 y el $80 \%$ ameritan atención, y los que tienen un porcentaje inferior al $50 \%$ se consideran (PINTO y SLEVIN, 1998 apud RABECHINI, 2007).

Aplicando la metodología de Slevin y Pinto (1987), los datos permitieron identificar los posibles errores del proyecto, desde el punto de vista de cada grupo de interés, y hacer recomendaciones. La identificación de los errores siguió la matriz de eficacia estratégica y táctica que se muestra del Cuadro 4.

Cuadro 4: Matriz de eficacia estratégica y táctica de un proyecto.

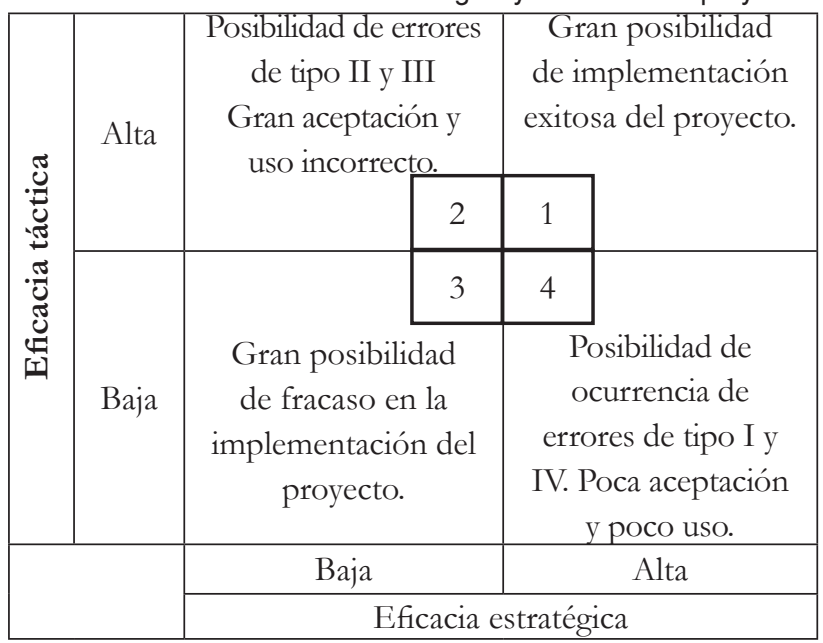

Fuente: Slevin y Pinto (1987, p. 37).

De acuerdo a la matriz, hay 4 (cuatro) tipos de combinaciones derivadas de la actuación de los gestores en el equilibrio entre la estrategia y la táctica durante el proceso. El error de tipo I se produce cuando se desarrolla un nivel inadecuado de actividades tácticas y se implementan pocas acciones a favor del proyecto. El error de tipo II se produce cuando se adopta una acción que no debería haber sido aplicada. El error de tipo III se produce cuando se resuelve el problema equivocado por medio de una acción eficaz. Por último, el error de tipo IV se produce cuando se es consciente de las acciones que deben perpetuarse, pero por alguna razón no se las perpetúa.

La principal limitación de este estudio es que los encuestados expresaron sus percepciones personales sobre el tema, que no representan necesariamente la posición oficial de las instituciones a las que pertenecen. Otra limitación se refiere a la poca participación de los miembros del G1.

\section{RESULTADOS Y DISCUSIÓN}

La Tabla 1 muestra los resultados de la moda y del porcentaje de éxito por factor crítico y por grupo en estudio. 
Tabla 1 - Moda y porcentaje de éxito por grupo.

\begin{tabular}{|c|c|c|c|c|c|c|c|c|c|c|c|c|c|c|c|c|}
\hline \multirow{2}{*}{$\begin{array}{l}\text { Factores } \\
\text { Críticos }\end{array}$} & \multicolumn{4}{|c|}{ G1 $(n=1)$} & \multicolumn{4}{|c|}{$\mathrm{G} 2(\mathrm{n}=6)$} & \multicolumn{4}{|c|}{ G3 $(n=13)$} & \multicolumn{4}{|c|}{$\mathrm{G} 4(\mathrm{n}=14)$} \\
\hline & Moda & $\begin{array}{c}\% \\
\text { Éxito }\end{array}$ & Mínimo & $\begin{array}{c}\% \\
\text { Éxito }\end{array}$ & Moda & $\begin{array}{c}\% \\
\text { Éxito }\end{array}$ & Mínimo & $\begin{array}{c}\% \\
\text { Éxito }\end{array}$ & Moda & $\begin{array}{c}\% \\
\text { Éxito }\end{array}$ & Mínimo & $\begin{array}{c}\% \\
\text { Éxito }\end{array}$ & Moda & $\begin{array}{c}\% \\
\text { Éxito }\end{array}$ & Mínimo & $\begin{array}{c}\% \\
\text { Éxito }\end{array}$ \\
\hline FCE 1 & 29 & $40 \%$ & 29 & $40 \%$ & 33 & $80 \%$ & 27 & $25 \%$ & 31 & $60 \%$ & 21 & $8 \%$ & 32 & $70 \%$ & 14 & $4 \%$ \\
\hline FCE 2 & 35 & $100 \%$ & 35 & $100 \%$ & 30 & $70 \%$ & 22 & $25 \%$ & & & & & & & & \\
\hline FCT 3 & 35 & $100 \%$ & 35 & $100 \%$ & 33 & $85 \%$ & 25 & $25 \%$ & 33 & $85 \%$ & 19 & $9 \%$ & 34 & $90 \%$ & 17 & $8 \%$ \\
\hline FCT 4 & 30 & $80 \%$ & 30 & $80 \%$ & 29 & $75 \%$ & 24 & $50 \%$ & & & & & & & & \\
\hline FCT 5 & 33 & $95 \%$ & 33 & $95 \%$ & 30 & $80 \%$ & 25 & $54 \%$ & & & & & & & & \\
\hline FCE 6 & 26 & $60 \%$ & 26 & $60 \%$ & 27 & $60 \%$ & 22 & $40 \%$ & & & & & & & & \\
\hline FCT 7 & 33 & $95 \%$ & 33 & $95 \%$ & 33 & $94 \%$ & 23 & $45 \%$ & 30 & $80 \%$ & 14 & $10 \%$ & 23 & $45 \%$ & 13 & $9 \%$ \\
\hline FCT 8 & 32 & $70 \%$ & 32 & $70 \%$ & 32 & $70 \%$ & 13 & $5 \%$ & 25 & $20 \%$ & 5 & $0 \%$ & 13 & $5 \%$ & 7 & $0 \%$ \\
\hline FCE 9 & 35 & $100 \%$ & 35 & $100 \%$ & 35 & $100 \%$ & 35 & $100 \%$ & 35 & $100 \%$ & 16 & $9 \%$ & 35 & $100 \%$ & 20 & $20 \%$ \\
\hline
\end{tabular}

Legenda: FCE: factor crítico estratégico; FCT: factor crítico táctico.

\section{Fuente: El autor.}

Los datos muestran que los grupos de interés estudiados eran heterogéneos. El análisis del G1 se vio perjudicado debido a que solo 1 (uno), de los 6 (seis) integrantes del grupo, devolvió el cuestionario. Aunque se mostró bastante receptivo al proyecto, el G2 consideró el FCE 1 (Objetivos) por debajo del $50 \%$ y el FCE 6 (Planificación) y FCT 8 (Clientes) entre el 50 y el $80 \%$. Todos los otros FCE y FCT funcionan bien (por arriba del $80 \%$ ).

El G2 es el único grupo en el que el porcentaje de la moda de todos los factores críticos están por arriba del $50 \%$. Los factores FCE 2 (Dirección), FCT 4 (Personal), FCE 6 (Planificación) y FCT 8 (Clientes) muestran valores dentro del rango entre el 50 y el $80 \%$, y requieren más atención por parte de los gestores del proyecto.

En el G3, los factores FCT 3 (Estructura orgánica), FCT 7 (Infraestructura) y FCT 9 (Apoyo al proyecto) están por arriba del $80 \%$. Sin embargo, el FCE 1 (Objetivos), con un $60 \%$, necesita más atención, mientras que el FCT 8 (Clientes), con un $20 \%$, se considera crítico.

El G4 muestra resultados similares a los del G3. Los factores FCT 3 y FCE 9 están por arriba del $80 \%$, mientras el FCE 1 (Objetivos), con un $70 \%$, necesita más atención, y el FCT 7 (Infraestructura), con un $45 \%$ y el FCT 8 (Clientes), con solo un $5 \%$, muestran resultados críticos.

Solo 2 (dos) factores críticos merecieron buenas opiniones en los 4 (cuatro) grupos: FCT 3 (Estructura orgánica) y FCE 9 (Apoyo al proyecto). Por otro lado, los factores que muestran las mayores diferencias de opinión son el FCE 1 (Objetivos) y FCT 7 (Infraestructura), lo que señala la gran heterogeneidad entre los grupos.

El G1, aunque confiando solamente en la opinión de 1 (uno) de los actores, muestra evidencia de falta de comprensión clara de los objetivos del CDAer (FCE 1). Esto requiere una intervención inmediata de los gestores, dado que los representantes de este grupo tienen un alto poder y sus decisiones afectan fundamentalmente el proyecto.
Por su parte, los representantes del G4 temen que los aspectos relacionados con el mantenimiento y la infraestructura (FCT 7) no se monitoreen adecuadamente. Esta percepción del G4 se enfrenta a las opiniones de los actores de los grupos G1, G2 y G3, que indican, respectivamente, un 95 , un 94 y un $80 \%$ de éxito. La discrepancia de las opiniones puede explicarse por la comunicación inadecuada de los gestores con los deportistas y usuarios de las instalaciones deportivas (representantes del G4) e indica un desequilibrio en el monitoreo entre los grupos de interés.

Esta afirmación se apoya en el análisis de los resultados del FCT 8 (Clientes), un factor que tuvo el peor desempeño en los grupos G2, G3 y G4, con los dos últimos mostrándose seriamente desinformados $(20$ y $5 \%$, respectivamente). Estas cifras indicarían que sus miembros se sienten excluidos del proceso de construcción del CDAer. Incluso el grupo de los gestores (G2) muestra solo un $70 \%$ de éxito para el FCT 8, cuando se esperaba al menos un $80 \%$. Aunque el proyecto está pasando de la fase de diseño a la de planificación, dentro de su ciclo de vida (SLEVIN; PINTO, 1987), este dato muestra que hay deficiencias en términos tácticos, que deben ser corregidas.

La diferencia entre los valores de las modas y los valores mínimos de cada factor crítico es el caso más probable de éxito que la implementación del proyecto va a encontrar. Esto se deduce que la moda representa el valor del mayor acuerdo entre todas las partes interesadas dentro de cada grupo, mientras que el valor mínimo representa la mayor insatisfacción posible, es decir, el peor de los casos. Cuanto más se acerquen los valores mínimos y las modas del $80 \%$, mayor será la homogeneidad entre los grupos de interés y, por consiguiente, menos probabilidades de conflicto de intereses.

La comparación entre los resultados de la moda y los valores mínimos para cada factor crítico evaluado por las partes interesadas se presenta en las Figuras 2, 3, 4 y 5. 
El G1, ya que solo tiene 1 (uno) evaluador, muestra los mismos valores en la moda y mínimo, lo que compromete el análisis de la heterogeneidad dentro de este grupo. Aún así, esta cifra muestra claramente el desequilibrio de la gestión del FCE 1 (Objetivos) y FCE 6 (Planificación), en comparación con los otros factores.

Figura 2 - Comparación de los factores críticos de éxito señalados por el G1, relativo a los resultados de las modas y los valores mínimos.

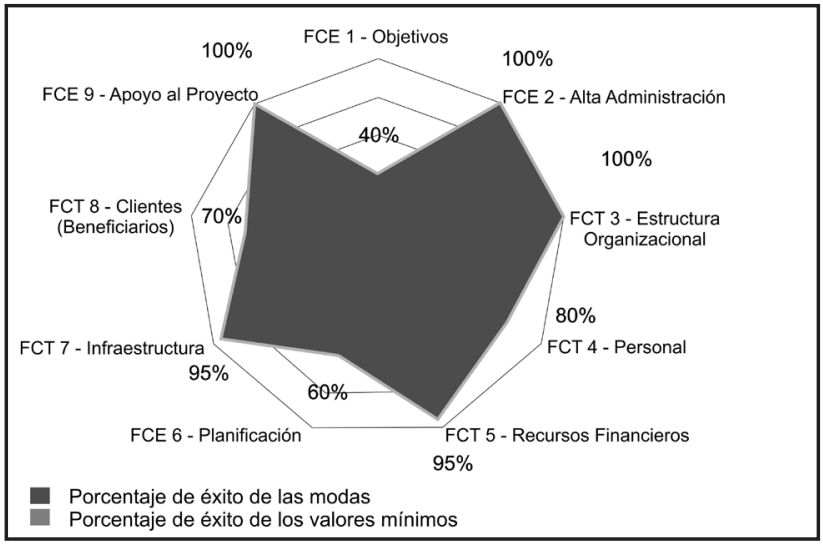

Fuente: El autor.

En la Figura 3 se puede observar que el FCT 8 (Clientes) es el factor crítico más heterogéneo para el G2, con una diferencia del $65 \%$. Otros factores, tales como el FCE 1 (Objetivos), FCE 2 (Dirección) y FCT 3 (Estructura orgánica), también deben recibir mayor atención, ya que la diferencia entre los valores de las modas y mínimos es superior al $45 \%$, es decir, muy heterogéneo.

Figura 3 - Comparación de los factores críticos de éxito señalados por el G2, relativo a los resultados de las modas y los valores mínimos.

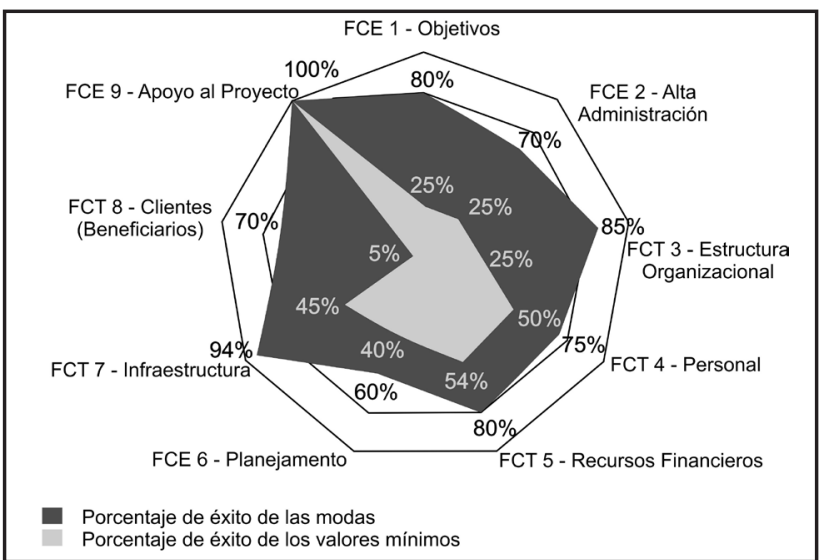

Fuente: El autor.

Los datos observados en las Figuras 4 y 5, que se refieren, respectivamente, al G3 y G4, muestran un panorama aún peor. La heterogeneidad de las respuestas señala grandes diferencias en todos los factores críticos en estudio, la mayor parte por arriba del $50 \%$. El peor caso es, una vez más, el del FCT 8 (Clientes), en el que tanto la moda como el valor mínimo son muy bajos, y todos los involucrados están de acuerdo.
Figura 4 - Comparación de los factores críticos de éxito señalados por el G3, relativo a los resultados de las modas y los valores mínimos.



Fuente: El autor.

Figura 5 - Comparación de los factores críticos de éxito señalados por el G4, relativo a los resultados de las modas y los valores mínimos.

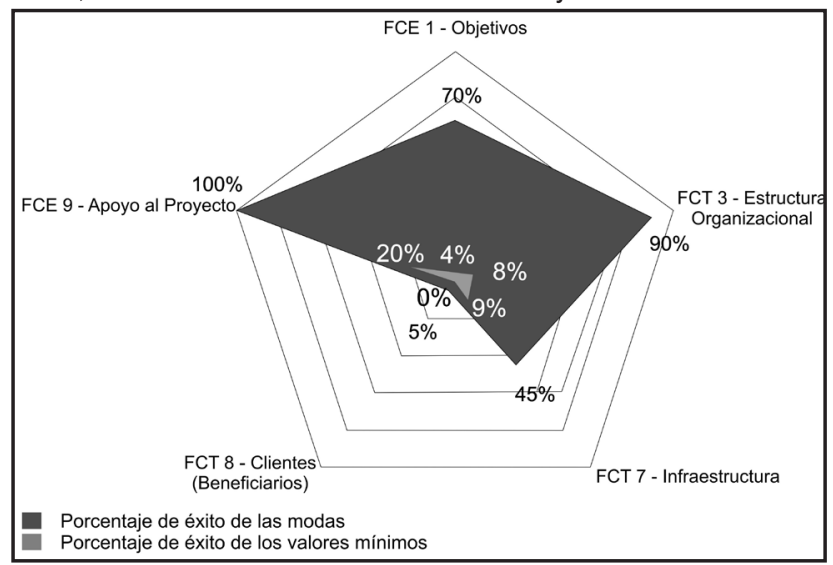

Fuente: El autor.

$\mathrm{El}$ análisis de este escenario sugiere que hay partes interesadas claramente insatisfechas en los grupos G3 y G4. De acuerdo con matriz de Qualman (1997), que se muestra en el Cuadro 1, estos actores representan la clase de los que tienen gran interés, pero poco poder. Su opinión no es suficiente para descarrilar el proyecto, pero aún así es importante que continúen asociados, ya que presentan ideas y ayudan con acciones menores. Esta interpretación se ve confirmada por el análisis de los resultados de la FCE 9 (Apoyo al proyecto), que cuenta con valores mínimos en torno al 9 y el $20 \%$ en los grupos G3 y G4, respectivamente. Esto sugiere que hay partes interesadas que no están alineados con la creación de CDAer, probablemente porque sus intereses no están siendo protegidos o porque la comunicación del proyecto no se está realizando correctamente.

En este sentido, aumenta la importancia de la matriz de eficacia estratégica y táctica, que se presenta en el Cuadro 5 , visto que el cruce de los datos relativos a las respuestas de cada grupo señala los aspectos que son más o menos eficaces y ayuda a identificar posibles errores de gestión. 
Cuadro 5 - Matriz de eficacia entre los aspectos estratégicos y tácticos.

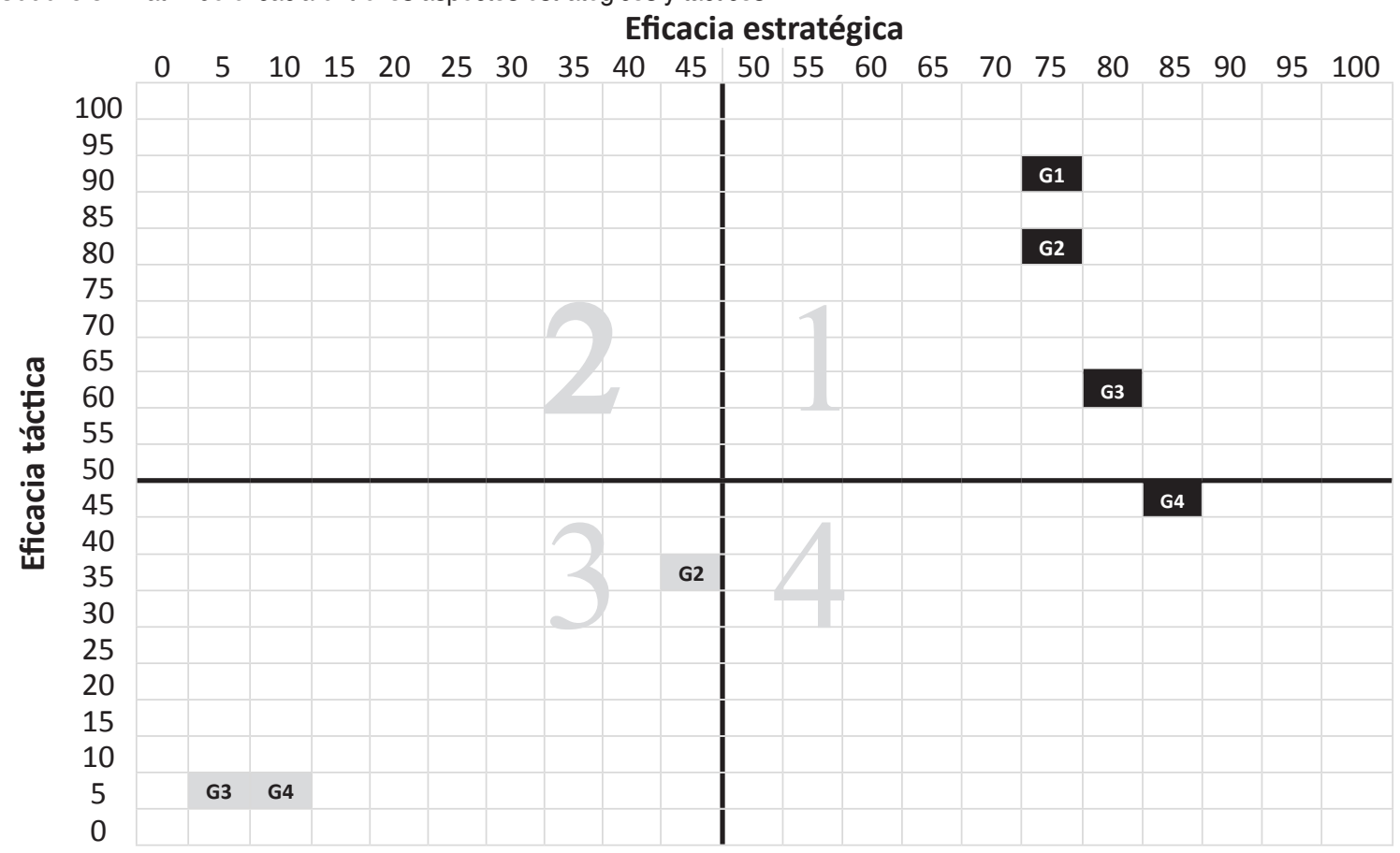

Legenda:

$\square$ Interpolación basada en los valores mínimos.

- Interpolación basada en las modas.

Obs.: en el G1, la moda y los valores mínimos son los mismos.

Fuente: El autor.

Mediante el análisis del cruce de los datos relativos a la moda, se observa claramente que, sumados, los factores críticos estratégicos (FCE) se insertan en el mismo rango de porcentaje de éxito, comprendido entre el 75 y el $85 \%$ (intervalo de 10 pp. pp.) Sin embargo, no se da lo mismo con los factores críticos tácticos (FCT), cuyo rango es mucho más amplio y está comprendido entre el 45 y el $95 \%$ (intervalo de 50 pp. pp.).

Otra observación inmediata se refiere al cuadrante en que se encuentra cada grupo de interés. Los grupos G1, G2 y G3 están en el cuadrante 1 que, según Slevin y Pinto (1997), muestra que tiene un gran potencial para la implementación exitosa. Por el contrario, el G4 está en el cuadrante 4 , zona probable de ocurrencia de errores de tipo I (proyecto con baja aceptación y baja eficiencia táctica) y tipo IV (baja iniciativa de corrección del curso del proyecto, aunque se conozcan las fallas).

En el estudio de los cruces de los datos relativos a los valores mínimos, se observa que el proyecto corre un gran riesgo de no ser implementado con éxito, puesto que las respuestas de 3 (tres) de los grupos en estudio se encuentran en el cuadrante 3. En este caso, como señalan Slevin y Pinto (1987), además de los errores de tipo I y tipo IV, también se debe prestar atención a errores tipo II (tomar medidas que no deberían haber sido adoptadas) y tipo III (impacto colateral de una medida correctiva, empleada para resolver un problema determinado, pero que afecta a otro).
Estos hallazgos son importantes y, aunque al parecer tengan un sesgo negativo, no todo está perdido. Rabechini (2007), aplicando la misma metodología, cita un estudio llevado a cabo en una empresa agroindustrial de Brasil, en el que los resultados fueron muy similares. El factor crítico Dirección fue uno de los que recibió los puntajes más altos, mientras que los factores Clientes y Comunicaciones necesitaron más atención y recibieron recomendaciones que culminaron en una reorientación de la gestión del proyecto. En tales casos, el autor sugiere que hay posibilidades factibles de intervención por parte de los gestores del proyecto.

\section{CONCLUSIÓN}

Este estudio tuvo por objeto analizar la forma en que los factores críticos de éxito, desde el punto de vista de las partes interesadas, ejercen impacto sobre la eficacia del proyecto de implantación del CDAer.

La metodología aplicada permitió concluir que los factores críticos de éxito, para la mayoría de los actores de los grupos G1, G2 y G3, responden a lo que se espera del proyecto que, a su vez, presenta una eficacia estratégica mayor que la táctica. El estudio también mostró que hay actores importantes (G4) que no están satisfechos, porque el proyecto carece de una estrategia de comunicación adecuada y de participación de estos actores en el proceso. El análisis de los valores mínimos también mostró los tipos de errores potenciales del proyecto, y presentó el problema de la heterogeneidad de opinión de las partes interesadas. 
Es posible derivar algunas lecciones de este estudio. En primer lugar, el entendimiento de que una gestión lejana de los principales interesados crea incertidumbre y produce una reducción en el apoyo a la realización de las actividades necesarias. En segundo lugar, implica que la concientización y el apoyo de las principales partes interesadas deben ser un factor importante en la estructuración de la propuesta de creación del CDAer, desde el principio. Por último, conlleva la realización de un diagnóstico claro de sus problemas, especialmente de los relacionados con los clientes, el personal, los recursos financieros y la estructura orgánica, respetando el equilibrio estratégico- táctico de la propuesta, desde el principio.

Teniendo en cuenta estas lecciones, se pueden hacer algunas recomendaciones para corregir el curso del proyecto. Destaca, entre ellas la necesidad de desarrollar un plan de comunicación, con el fin de mejorar el intercambio de información entre los gestores y los diferentes grupos de interés.

\section{REFERENCIAS}

BRASIL. Comando da Aeronáutica. Comissão de Desportos da Aeronáutica. Proposta de criação do Centro de Desportos da Aeronáutica (CDAer). Ofício no 10/VP/614, de 17/03/2015. Rio de Janeiro, 2015.

FREEMAN, R.E. Strategic management: A stakeholder approach. Boston: Pitman, 1984.

GOLDSCHIMIDT, A. Stakeholders: como interagir com tantos públicos diferentes. Revista RETS, 2005. Disponível em: <https://www.google.com.br/url?sa=t\&rct=j $\& q=\& e s r c=s \&$ source $=$ web\& $c d=2 \&$ ved $=0$ CCUQFjAB\&url= http\%3A\%2F\%2Fwww.novosolhos.com.br\%2Fdownload. php\%3Fextensa0\%3Drtf\%26original\%3DStakeholde rs\%2520-\%2520\%2520Partes\%2520Interessadas\% 2520RETS\%2520ago2005.rt\%26servidor\%3Darq material\%2F59_58.rtf\&ei=H0zYU4SQLIfhsATOioKwDA\& usg=AFQjCNE901K7sZzEWXbTQ36v0KJ8pdcLaA\&sig2 =Y-o7eMV85hacis6tXENabg>. Acesso em: 26 jul. 2014.

MARCONI, M. A; LAKATOS, E. M. Fundamentação da metodologia científica. 5.ed. São Paulo: Atlas, 2003. 311p.

MOTTA, J. R. G. O negócio das arenas: profissionalismo esportivo, cultura e entretenimento. Future Studies Researsh Journal. São Paulo, v. 4, n. 2, p. 21-48, jul./dez. 2012.

PINTO, J. K.; SLEVIN, D.P. Critical success factors in effective project implementation. Sloan Management Review, 1987. Disponível em: <http://gspa.grade. nida.ac.th/pdf/PA\%20780\%20(Pakorn)/8.Critical\%20 Success\%20Factors\%20in\%20Effective\%20 Project\%20Implementati.pdf>. Acesso em: 24 jul. 2014.
Otra sugerencia es que se debe preparar y establecer un proceso de apoyo a los clientes y beneficiarios del CDAer, tratando de involucrarlos en el proyecto y mejorar el alcance del plan de implementación que se ha preparado. Se asocia también la necesidad de identificar las razones por las que algunos actores no apoyan la creación de CDAer y, en consecuencia, trazar un plan de contingencia para garantizar una reorientación adecuada de las acciones a implementar.

Además, se sugiere aumentar la eficacia táctica del proyecto mediante el mapeo y detalle de las funciones del personal y los recursos que se emplearán en el CDAer. Por último, aunque el análisis de los datos del G1 se haya hecho sobre las respuestas de solo 1 (uno) evaluador, se debe tener en cuenta que este grupo está formado por los actores más poderosos, ya que son los responsables de las decisiones finales relativas al proyecto. Por lo tanto, el acercamiento y la búsqueda de orientación de estos actores deben ser una constante en todo el proceso.
QUALMAN, A. Note on stakeholder analysis. NGO Connect, 1997. Disponível em:<http://www. ngoconnect.net/documents/592341/749044/A+Note+ on+Stakeholder+Analysis>. Acesso em: 28 jul. 2014.

RABECHINI JR, R. O gerente de projetos na empresa. 2. ed. São Paulo: Atlas, 2007. 210p.

ROBINSON et al. Organising an Olympic Sport Organization. In: CAMY J.; ROBINSON, L. (Org). Managing Olympic Sports Organisations. Lausanne: Olympic Solidarity, p. 1-60, 2007.

ROCHA, C. M; BASTOS, F. C. Gestão do esporte: definindo a área. Rev. Bras. Educ. Fís. Esporte. São Paulo, v. 25, p. 91-103, dez. 2011.

SANTOS, F. R. O emprego da análise de stakeholders em um plano estratégico para a gestão da mobilidade sustentável: estudo de caso do campus da Universidade de Brasília. 2008. 99 f. Dissertação (Mestrado em Transportes) - Departamento de Engenharia Civil e Ambiental, Universidade de Brasília, Brasília, 2008.

SLEVIN, D. P.; PINTO, J.K. Balancing strategy and tatics in project implementation. Sloan Management Review, v. 29, n. 6, p. 33-41, 1987.

TOLEDO, J. R. Campeonato do índice de confiança social: Forças Armadas continuam do G4 e partidos seguem firme na lanterna. Forças Terrestres. Rio de Janeiro, Ago. 2013. Disponível em:<http://www. forte.jor.br/2013/08/01/campeonato-do-indice-deconfianca-social-forcas-armadas-continuam-no-g4e-partidos-seguem-firmes-na-lanterna/>. Acesso em: 27 jul. 2014. 